\title{
ERRATUM
}

\section{From Gestures to Words: Verbal labelling of hand movements reduces activation of the inferior frontal gyrus-Erratum}

\author{
M. Miyahara, R. Kitada, A. Sasaki, Y. Okamoto, H.C. Tanabe, AND N. Sadato \\ doi:10.1017/S1355617711001172, Published by Cambridge University Press 5 August 2011. \\ The following Abstract on page 8 of the Final Program of the INS Mid-Year Meeting in Auckland, NZ was inadvertently listed \\ as "Withdrawn" from Presentation. Indeed, this abstract was presented on Friday, July 8, 2011.
}

The INS mistakenly believed that the author was absent from the Mid-Year meeting and thus the Withdrawn banner was placed on his abstract. He in fact did attend and present his abstract on Friday, July 8, 2011 in Auckland, NZ. This abstract is presented below in its entirety.

We apologize to the author for this error.

\section{CORRECTION TO THE ADDENDUM TO THE FINAL PROGRAM OF THE INS Mid-Year MEETING, July 6-9, 2011 Auckland, New Zealand}

Objective: Verbal labeling (VL) is known as an efficient mnemonic for visuospatial working memory. However, little is known about the neuronal basis of the process. This study aimed to identify the neural substrates involved in the effect of VL on increasingly complex sequential memory for hand movements.

Participants and Methods: We used fMRI to test our hypothesis that VL would keep neuronal activity in motor related regions constant for the memory, regardless of movement complexity. Sixteen young healthy adults performed an immediate imitation task, a modified version of the Kaufman Hand Movement Test (Kaufman \& Kaufman, 1983) consisting of six levels of increasingly complexity. After the fMRI experiment, the subjects reported in which complexity level they had formed verbal labels. Based on the report, we classified individual conditions into with VL (VL+) or without VL (VL-) at each complexity level.

Results: As compared to VL + condition, VL - condition activated the inferior frontal gyrus, middle frontal gyrus, precentral gyrus and angular gyrus, whereas the opposite contrast produced no significant brain regions. Within the brain regions activated by $\mathrm{VL}$ - condition relative to VL+ condition, there was increasing activity with progressive complexity in the inferior frontal gyrus under VL - condition, whereas in the VL+ condition, the activity of the same regions was constantly low, regardless of movement complexity.

Conclusions: VL is an efficient heuristic, reducing the cost of cortical activation in the inferior frontal gyrus. Correspondence: Motohide Miyahara, PhD, University of Otago, School of Physical Education, University of Otago, PO Box 56, Dunedin 9054, New Zealand. E-mail: motohide.miyahara@otago.ac.nz

\section{REFERENCE}

Miyahara, M., Kitada, R., Sasaki, A., Okamoto, Y., Tanabe, H.C., \& Sadato, N. (2011). From Gestures to Words: Verbal labelling of hand movements reduces activation of the inferior frontal gyrus. Journal of the International Neuropsychological Society, 17, Supplement 2, 8. doi: $10.1017 /$ S1355617711001172. 